NASA Technical Memorandum 105257

ICOMP-91- 18

\title{
Multigrid Calculation of Three-Dimensional Viscous Cascade Flows
}

A. Arnone

University of Florence

Florence, Italy

and Institute for Computational Mechanics in Propulsion

Lewis Research Center

Cleveland, Ohio

M.-S. Liou and L.A. Povinelli

Lewis Research Center

Cleveland, Ohio

(NASA-TM-105257) MULTIGRID CALCULATION OF

THREE-DIMENSIONAL VISCOUS CASCADE FLOWS

(NASA) $25 \mathrm{p}$
CSCL $12 A$ 


\title{
MULTIGRID CALCULATION OF THREE-DIMENSIONAL VISCOUS CASCADE FLOWS
}

\author{
A. Arnone ${ }^{*}$ \\ University of Florence \\ Florence, Italy \\ and Institute for Computational Mechanics in Propulsion \\ Lewis Research Center \\ Cleveland, Ohio 44135 \\ M.-S. Liou ${ }^{* *}$ and L.A. Povinelli ${ }^{+}$ \\ National Aeronautics and Space Administration \\ Lewis Research Center \\ Cleveland, Ohio 44135
}

\begin{abstract}
$\underline{\text { Abstract }}$
A three-dimensional code for viscous cascade flow prediction has been developed. The space discretization uses a cell-centered scheme with eigenvalue scaling to weigh the artificial dissipation terms. Computational efficiency of a four-stage Runge-Kutta scheme is enhanced by using variable coefficients, implicit residual smoothing, and a full-multigrid method. The Baldwin-Lomax eddy-viscosity model is used for turbulence closure. A zonal, non-periodic grid is used to minimize mesh distortion in and downstream of the throat region. Applications are presented for an annular vane with and without end wall contouring, and for a large-scale linear cascade. The calculation is validated by comparing with experiments and by stud;ing grid dependency.
\end{abstract}

\section{Introduction}

Improving efficiency and specific work while reducing weight, cost, and number of components and maintaining a good level of performance in a wide range of operational conditions is the goal of turbomachinery design. The last decade has seen an impressive evolution both in the understanding and in the simulation of flow features. In this process Computational Fluid Dynamics (CFD) is playing a more and more important role. In turbomachinery applications, modern components operate under very complex three-dimensional flow conditions, and futher improvement of performance requires detailed knowledge of the flow structure. Particularly, the need to predict off-design conditions, secondary flows, and heat transfer forces us to look at viscous models. Even if a two-dimensional or quasi threedimensional analysis is very useful, three-dimensional simulation will be the basic tool in the

\footnotetext{
* Assistant Professor, Department of Energy Engineering

** Senior Scientist, Internal Fluid Mechanics Division, Member AJAA

+ Deputy Chief, Internal Fluid Mechanics Division, Member AIAA
} 
design of the next generation of turbomachinery. The real flow inside a turbomachine is unsteady and dominated by rotor-stator interactions and wake effects. However, the timeaccurate simulation of an entire turbine or compressor is beyond the capability of current computers. On the contrary, a steady, viscous, blade-row analysis is now beginning to be feasible for designers, although much effort is still needed to improve accuracy and to reduce the computational cost. The works of Chima ${ }^{1}$, Weber ${ }^{2}, \mathrm{Hah}^{3}$, Nakahashi ${ }^{4}$, Choi ${ }^{5}$, Dawes ${ }^{6}$, and Subramanian ${ }^{7}$ are some important examples of three-dimensional viscous cascade flow predictions.

In 1988, the University of Florence started a joint project with NASA (ICASE, and ICOMP) on viscous cascade flow simulation. During this research project, we developed the TRAF2D code (TRAnsonic Flow 2D) ${ }^{8.9}$. This code is capable of solving two-dimensional viscous cascade flows using $\mathrm{H}$-type or C-type grids and of predicting heat transfer effects. In the present paper the procedure is extended to the three-dimensional case (TRAF3D). Particular attention has been dedicated to aspects which are important for the designer, such as accuracy, computational cost, and the correct prediction of pressure, exit angles, and loss coefficients.

As for accuracy, a new kind of elliptically-generated C-type grid is stacked in three dimensions. The removal of periodicity on the wake allows the grid to be only slightly distorted even for cascades having a large camber or a high stagger angle. This allows us to pick up details of the throat flow with a reasonable number of grid points. In addition, a very low level of artificial dissipation is guaranteed by eigenvalues scaling, which is a three-dimensional extension of the one proposed by Martinelli ${ }^{10}$, and Swanson and Turkel ${ }^{11}$.

As for efficiency, the Reynolds-averaged Navier-Stokes equations are solved using a Runge-Kutta scheme in conjunction with accelerating techniques. Variable-coefficient implicit residual smoothing, as well as the Full-Approximation-Storage multigrid scheme of Brandt and Jameson have been used in the TRAF3D code. Those accelerating strategies are implemented in conjunction with grid refinement to get a Full Multigrid Method. The two-layer eddy-viscosity model of Baldwin and Lomax is used for the turbulence closure.

The capability of the code is shown by comparing the computed results to experiments for the Goldman annular vane with and without end wall contouring, and for the low speed Langston linear cascade. For the case of the vane, a grid dependency analysis is presented.

By using the accelerating strategies, detailed, viscous 3D solutions on a grid with nearly half a million points can be obtained in less than one hour on a modern supercomputer such as a Cray Y-MP.

\section{Governing Equations}

Let $\rho, u, v, w, p, T, E$, and $H$ denote respectively density, the velocity components in the $x, y$, and $z$ Cartesian directions, pressure, temperature, specific total energy, and specific total enthalpy. The three-dimensional, unsteady, Reynolds-averaged Navier-Stokes equations, neglecting body forces and heat sources, can be written for a fixed blade passage in conservative form in a curvilinear coordinate system $\xi, \eta, \zeta$ as,

$$
\frac{\partial\left(J^{\prime} Q\right)}{\partial t}+\frac{\partial F}{\partial \xi}+\frac{\partial G}{\partial \eta}+\frac{\partial H}{\partial \zeta}=\frac{\partial F_{v}}{\partial \xi}+\frac{\partial G_{v}}{\partial \eta}+\frac{\partial H_{v}}{\partial \zeta}
$$


where,

$$
\begin{gathered}
Q=\left\{\begin{array}{l}
\rho \\
\rho u \\
\rho v \\
\rho w \\
\rho E
\end{array}\right\}, \quad F=J^{I}\left\{\begin{array}{l}
\rho U \\
\rho u U+\xi_{x} p \\
\rho v U+\xi_{y} p \\
\rho w U+\xi_{p} p \\
\rho H U
\end{array}\right\} \\
G=J^{I}\left\{\begin{array}{l}
\rho V \\
\rho u V+\eta_{x} p \\
\rho v V+\eta_{y} p \\
\rho w V+\eta_{z} p \\
\rho H V
\end{array}\right\}, H=J^{-1}\left\{\begin{array}{l}
\rho W \\
\rho u W+\zeta_{x} p \\
\rho v W+\zeta_{y} p \\
\rho w W+\zeta_{z} p \\
\rho H W
\end{array}\right\}
\end{gathered}
$$

The contravariant velocity components of eqs. (2) are written as,

$$
\begin{gathered}
U=\xi_{x} u+\xi_{y} v+\xi_{z} w \\
V=\eta_{x} u+\eta_{y} v+\eta_{z} w \\
W=\zeta_{x} u+\zeta_{y} v+\zeta_{z} w
\end{gathered}
$$

and the transformation metrics are defined by,

$$
\begin{aligned}
& \xi_{x}=J\left(y_{\eta} z^{-y_{\zeta^{z}}}\right) \\
& \xi_{y}=J\left({ }^{z} \eta^{x} \zeta^{-z} \zeta^{x} \eta\right) \\
& \xi_{z}=J\left(x_{\eta} y_{\zeta}-x_{\zeta} y_{\eta}\right) \\
& \eta_{x}=J\left(y_{\zeta}^{z}-y_{\xi}^{z} \zeta\right) \\
& \eta_{y}=J\left(z_{\zeta}{ }^{x_{\xi}}-z_{\xi} x_{\zeta}\right) \\
& \eta_{z}=J\left(x_{\zeta} y_{\xi}-x_{\xi} y_{\zeta}\right) \\
& \zeta_{x}=J\left(y_{\xi^{z}}{ }^{z}-z_{\xi}{ }^{y_{\eta}}\right) \\
& \zeta_{y}=J\left(z_{\xi} x_{\eta}-x_{\xi^{z}}{ }_{\eta}\right) \\
& \zeta_{z}=J\left(x_{\xi} y_{\eta}-y_{\xi} x_{\eta}\right)
\end{aligned}
$$

where the Jacobian of the transformation $J$ is,

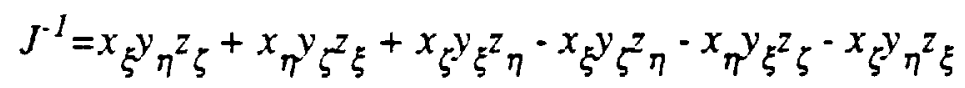

The viscous flux terms are assembled in the form,

$$
F_{v}=J^{-1}\left\{\begin{array}{c}
0 \\
\xi_{x} \tau_{x x}+\xi_{y} \tau_{x y}+\xi_{z} \tau_{x z} \\
\xi_{x} \tau_{y x}+\xi_{y} \tau_{y y}+\xi_{z} \tau_{y z} \\
\xi_{x} \tau_{z x}+\xi_{y} \tau_{z y}+\xi_{z} \tau_{z z} \\
\xi_{x} \beta_{x}+\xi_{y} \beta_{y}+\xi_{z} \beta_{z}
\end{array}\right\} G_{v}=J^{-1}\left\{\begin{array}{c}
0 \\
\eta_{x} \tau_{x x}+\eta_{y} \tau_{x y}+\eta_{z} \tau_{x z} \\
\eta_{x} \tau_{y x}+\eta_{y} \tau_{y y}+\eta_{z} \tau_{y z} \\
\eta_{x} \tau_{z x}+\eta_{y} \tau_{z y}+\eta_{z} \tau_{z z} \\
\eta_{x} \beta_{x}+\eta_{y} \beta_{y}+\eta_{z} \beta_{z}
\end{array}\right\} H_{v}=J^{-1}\left\{\begin{array}{c}
0 \\
\zeta_{x} \tau_{x x}+\zeta_{y} \tau_{x y}+\zeta_{z} \tau_{x z} \\
\zeta_{x} \tau_{y x}+\zeta_{y} \tau_{y y}+\zeta_{z} \tau_{y z} \\
\zeta_{x} \tau_{z x}+\zeta_{y} \tau_{z y}+\zeta_{z} \tau_{z z} \\
\zeta_{x} \beta_{x}+\zeta_{y} \beta_{y}+\zeta_{z} \beta_{z}
\end{array}\right\}
$$


where,

$$
\begin{aligned}
& \tau_{x x}=2 \mu u_{x}+\lambda\left(u_{x}+v_{y}+w_{z}\right) \\
& \tau_{y y}=2 \mu v_{y}+\lambda\left(u_{x}+v_{y}+w_{z}\right) \\
& \tau_{z z}=2 \mu w_{z}+\lambda\left(u_{x}+v_{y}+w_{z}\right) \\
& \tau_{x y}=\tau_{y x}=\mu\left(u_{y}+v_{x}\right) \\
& \tau_{x z}=\tau_{z x}=\mu\left(u_{z}+w_{x}\right) \\
& \tau_{y z}=\tau_{z x}=\mu\left(v_{z}+w_{y}\right) \\
& \beta_{x}=u \tau_{x x}+v \tau_{x y}+w \tau_{x z}+k T_{x} \\
& \beta_{y}=u \tau_{y x}+v \tau_{y y}+w \tau_{y z}+k T_{y} \\
& \beta_{z}=u \tau_{z x}+v \tau_{z y}+w \tau_{z z}+k T_{z}
\end{aligned}
$$

and the Cartesian derivatives of (7) are expressed in terms of $\xi_{-}, \eta_{-}$, and $\zeta$-derivatives using the chain rule, i.e.,

$$
u_{x}=\xi_{x} u_{\xi}+\eta_{x} u_{\eta}+\zeta_{x} u_{\zeta}
$$

The pressure is obtained from the equation of state,

$$
p=\rho R T
$$

According to the Stokes hypothesis $\lambda$ is taken to be $-2 \mu / 3$ and a power law is used to determine the molecular coefficient of viscosity $\mu$ as function of temperature. The eddy-viscosity hypothesis is used to account for the effect of turbulence. The molecular viscosity $\mu$ and the molecular thermal conductivity $k$ are replaced with,

$$
\begin{gathered}
\mu=\mu_{l}+\mu_{l} \\
k=c_{p}\left[\left(\frac{\mu}{P r}\right)_{l}+\left(\frac{\mu}{P r}\right)_{l}\right]
\end{gathered}
$$

where $c_{p}$ is the specific heat at constant pressure, $P r$ is the Prandtl number, and the subscripts $l$ and $t$ refer to laminar and turbulent respectively. The turbulent quantities $\mu_{t}$ and $\mathrm{Pr}_{t}$ are computed using the two-layer mixing length model of Baldwin and Lomax ${ }^{12}$. The contribution of the eddy viscosity is computed separately in the blade-to-blade direction $\eta$ and in the spanwise direction $\zeta$. The inverse of the square of the wall distances $d$ is then used to compute the resulting eddy viscosity, 


$$
\begin{gathered}
f=\frac{11 d_{\eta}^{2}}{: 11 d_{\eta}^{2}+11 d_{\zeta}^{2}} \\
\mu_{t}=f\left(\mu_{t}\right)_{\eta}+(1-f)\left(\mu_{t}\right)_{\zeta}
\end{gathered}
$$

The transitional criteria of Baldwin and Lomax is adopted on the airfoil surface while on the end walls the shear layer is assumed to be fully-turbulent from the inlet boundary.

\section{Spatial Discretization}

Traditionally, using a finite-volume approach, the goveming equations are discretized in space starting from an integral formulation and without any intermediate mapping. The transformation metrics of (4) can be then associated with the projections of the face areas as the contravariant components of (3) can be related to the normal components of velocity. In the present work, due to the large use of eigenvalues and curvilinear quantities, we found it more convenient to map the Cartesian space $(x, y, z)$ in a generalized curvilinear one $(\xi, \eta, \zeta)$. In the curvilinear system, the equation of motion (1) can be easily rewritten in integral form by means of Green's theorem and the metric terms are handled following the standard finite-volume formulation. The computational domain is divided into hexahedrons and the transformation metrics are evaluated so that the projected areas of the cell-faces are given by the ratio of the appropriate metric derivatives to the Jacobian ones, i.e. $\xi_{x} / J$ is the projection onto the $x$-axis of a cell face at a fixed $\xi$ location. A cell-centered scheme is used to store the flow variables. On each cell face the convective and diffusive fluxes are calculated after computing the necessary flow quantities at the face center. Those quantities are obtained by a simple averaging of adjacent cell-center values of the dependent variables.

\section{Boundary Conditions}

It is well known that, when dealing with a time-marching formulation, the reflecting behavior of the numerical boundary conditions must be minimized in order to enhance the convergence rate to the steady state. In cascade calculations we have four different types of boundaries: inlet, outlet, solid walls, and periodicity. According to the theory of characteristics, the flow angles, total pressure, and total enthalpy are specified at the subsonic-axial inlet, while the outgoing Riemann invariant is taken from the interior. The presence of an inlet boundary layer, on hub and tip end walls, is accounted for by giving a total pressure and a total temperature profile whose distribution simulates the experimental one. At the subsonic-axial outlet, the average value of the static pressure at the hub is prescribed and the density and components of velocity are extrapolated together with the circumferential distribution of pressure. The radial equilibrium equation is used to determine the spanwise distribution of the static pressure. On 
solid walls, the momentum equation, the no-slip condition, and the temperature condition are used to compute pressure and density. For the calculations presented in this paper, all the walls have been assumed to be adiabatic.

Cell-centered schemes are generally implemented using phantom cells to handle the boundaries. The periodicity from blade passage to blade passage is, therefore, easily overimposed by setting periodic phantom cell values. On the wake, where the grid is not periodic, the phantom cells overlap the real ones. Linear interpolations are then used to compute the value of the dependent variables in phantom cells.

\section{Artificial Dissipation}

In viscous calculations, dissipative properties are present due to diffusive terms. As well experienced away from the shear layer regions, the physical diffusion is generally not sufficient to prevent the odd-even point decoupling of centered schemes. Thus, to maintain stability and to prevent oscillations near shocks or stagnation points, artificial dissipation terms are also included in the viscous calculations. Equation (1) is written in semi-discrete form as,

$$
\frac{\partial Q}{\partial t}+C(Q)-D(Q)=0
$$

where the discrete operator $C$ accounts for the physical convective and diffusive terms, while $D$ is the operator for the artificial dissipation. The artificial dissipation model used in this paper is basically the one originally introduced by Jameson, Schmidt, and Turkel ${ }^{13}$. In order to minimize the amount of artificial diffusion inside the shear layer, the eigenvalues scaling of Martinelli ${ }^{10}$, and Swanson and Turkel ${ }^{11}$ have been used to weight these terms. The quantity $D(Q)$ in eq. (14) is defined as,

$$
D(Q)=\left(D_{\xi}^{2}-D_{\xi}^{4}+D_{\eta}^{2}-D_{\eta}^{4}+D_{\zeta}^{2}-D_{\zeta}^{4}\right) Q
$$

where, for example, in the $\xi$ curvilinear coordinates we have,

$$
\begin{aligned}
& D_{\xi}^{2} Q=\nabla_{\xi}\left(\Lambda_{i+1 / 2, j, k} \varepsilon_{i+1 / 2, j, k}^{(2)}\right) \Delta_{\xi} Q_{i, j, k} \\
& D_{\xi}^{4} Q=\nabla_{\xi}\left(\Lambda_{i+1 / 2, j, k} \varepsilon_{i+1 / 2, j, k}^{(4)}\right) \Delta_{\xi} \nabla_{\xi} \Delta_{\xi} Q_{i, j, k}
\end{aligned}
$$

$i, j, k$ are indices associated with the $\xi, \eta, \zeta$ directions and $\Delta_{\xi}, \nabla_{\xi}$ are forward and backward difference operators in the $\xi$ direction. Following the works of refs. 10 and 11 , the variable scaling factor $\Lambda$ is defined for the three-dimensional case as, 


$$
\Lambda_{i+l / 2, j, k}=\frac{1}{2}\left[\left(\Lambda_{\xi}\right)_{i, j, k}+\left(\Lambda_{\xi}\right)_{i+l, j, k}\right]
$$

where,

$$
\Lambda_{\xi}=\phi_{\xi} \lambda_{\xi}
$$

The definition of the coefficient $\phi$ has been extended to the three-dimensional case as follows,

$$
\begin{aligned}
& \phi_{\xi}=1+\left(\frac{\lambda_{\eta}}{\lambda_{\xi}}\right)^{\sigma}+\left(\frac{\lambda_{\zeta}}{\lambda_{\xi}}\right)^{\sigma} \\
& \phi_{\eta}=1+\left(\frac{\lambda_{\xi}}{\lambda_{\eta}}\right)^{\sigma}+\left(\frac{\lambda_{\zeta}}{\lambda_{\eta}}\right)^{\sigma} \\
& \phi_{\zeta}=1+\left(\frac{\lambda_{\xi}}{\lambda_{\zeta}}\right)^{\sigma}+\left(\frac{\lambda_{\eta}}{\lambda_{\zeta}}\right)^{\sigma}
\end{aligned}
$$

where $\lambda_{\xi}, \lambda_{\eta}$, and $\lambda_{\zeta}$ are the scaled spectral radii of the flux Jacobian matrices for the convective terms,

$$
\begin{aligned}
& \lambda_{\xi}=|U|+a \sqrt{\xi_{x}^{2}+\xi_{y}^{2}+\xi_{z}^{2}} \\
& \lambda_{\eta}=|V|+a \sqrt{\eta_{x}^{2}+\eta_{y}^{2}+\eta_{z}^{2}} \\
& \lambda_{\zeta}=/ W /+a \sqrt{\zeta_{x}^{2}+\zeta_{y}^{2}+\zeta_{z}^{2}}
\end{aligned}
$$

and $a$ is the speed of sound. The exponent $\sigma$ is generally defined by $0<\sigma \leq 1$, and for twodimensional applications, the value of $2 / 3$ gives satisfactory results. In three-dimensional cascade flow calculations, we generally have highly stretched meshes in two directions near corners. We found that $\sigma=0.4$ introduces enough scaling without compromising the robustness. The coefficients $\varepsilon^{(2)}$ and $\varepsilon^{(4)}$ use the pressure as a sensor for shocks and stagnation points, and are defined as follows,

$$
\varepsilon_{i+1 / 2, j, k}^{(2)}=K^{(2)} \operatorname{MAX}\left(v_{i-1, j, k}, v_{i, j, k}, v_{i+1, j, k}, v_{i+2, j, k}\right)
$$




$$
\begin{aligned}
v_{i, j, k}= & \left|\frac{p_{i \cdot I, j, k}-2 p_{i, j, k}+p_{i+1, j, k}}{p_{i-l, j, k}+2 p_{i, j, k}+p_{i+1, j, k}}\right| \\
& ; \\
\varepsilon_{i+1 / 2, j, k}^{(4)}= & \operatorname{MAX}\left[0,\left(K^{(4)}-\varepsilon_{i+1 / 2, j, k}^{(2)}\right)\right]
\end{aligned}
$$

where typical values for the constants $K^{(2)}$ and $K^{(4)}$ are $1 / 2$ and $1 / 64$ respectively. For the remaining directions $\eta$ and $\zeta$, the contribution of dissipation is defined in a similar way. The computation of the dissipative terms is carried out in each coordinate direction as the difference between first and third difference operators. Those operators are set to zero on solid walls in order to reduce the global error on the conservation property and to prevent the presence of undamped modes ${ }^{11,14,15}$.

\section{Time-Stepping Scheme}

The system of the differential equation (14) is advanced in time using an explicit four stage Runge-Kutta scheme until the steady-state solution is reached. A hybrid scheme is implemented, where, for economy, the viscous terms are evaluated only at the first stage and then frozen for the remaining stages. If $n$ is the index associated with time we will write it in the form,

$$
\begin{gathered}
Q^{(0)}=Q^{n} \\
Q^{(1)}=Q^{(0)}+\alpha_{1} \Delta t R\left(Q^{(0)}\right) \\
Q^{(2)}=Q^{(0)}+\alpha_{2} \Delta t R\left(Q^{(1)}\right) \\
Q^{(3)}=Q^{(0)}+\alpha_{3} \Delta t R\left(Q^{(2)}\right) \\
Q^{(4)}=Q^{(0)}+\alpha_{4} \Delta t R\left(Q^{(3)}\right) \\
Q^{n+1}=Q^{(4)} \\
\alpha_{1}=\frac{1}{4}, \quad \alpha_{2}=\frac{1}{3}, \quad \alpha_{3}=\frac{1}{2}, \quad \alpha_{4}=1
\end{gathered}
$$

where the residual $R(Q)$ is defined by,

$$
R(Q)=\Delta t J[C(Q)-D(Q)]
$$

Good, high-frequency damping properties, important for the multigrid process, have been obtained by performing two evaluations of the artificial dissipative terms, at the first and second stages. It is worthwhile to notice that, in the Runge-Kutta time-stepping schemes, the steady state solution is independent of the time step; therefore, this stepping is particularly amenable to convergence acceleration techniques. 


\section{Acceleration Techniques}

In order to reduce the computational cost, four techniques are employed to speed up convergence to the steady state-solution. These techniques: 1) local time-stepping; 2) residual smoothing; 3 ) multigrid; 4) grid refinement; are separately described in the following.

\section{Local Time-Stepping}

For steady state calculations with a time-marching approach, a faster expulsion of disturbances can be achieved by locally using the maximum available time step. In the present work the local time step limit $\Delta t$ is computed accounting for both the convective $\left(\Delta t_{c}\right)$ and diffusive $\left(\Delta t_{d}\right)$ contributions as follows,

$$
\Delta t=c_{0}\left(\frac{\Delta t_{c} \Delta t_{d}}{\Delta t_{c}+\Delta t_{d}}\right)
$$

where $c_{0}$ is a constant usually taken to be the Courant-Friedrichs-Lewy (CFL) number. Specifically, for the inviscid and viscous time step we used,

$$
\begin{gathered}
\Delta_{c}=\frac{1}{\lambda_{\xi}+\lambda_{\eta}+\lambda_{\zeta}} \\
\Delta_{d}=\frac{1}{K \frac{\gamma \mu}{\rho P r} J^{2}\left(s_{\eta}^{2} s_{\zeta}^{2}+S_{\xi}^{2} S_{\zeta}^{2}+S_{\xi}^{2} s_{\eta}^{2}\right)}
\end{gathered}
$$

where $\gamma$ is the specific heat ratio and,

$$
S_{\xi}^{2}=x_{\xi}^{2}+y_{\xi}^{2}+z_{\xi}^{2}, \quad S_{\eta}^{2}=x_{\eta}^{2}+y_{\eta}^{2}+z_{\eta}^{2}, \quad S_{\zeta}^{2}=x_{\zeta}^{2}+y_{\zeta}^{2}+z_{\zeta}^{2}
$$

$K_{t}$ being a constant whose value has been set equal to 2.5 based on numerical experiments.

\section{Residual smoothing}

An implicit smoothing of residuals is used to extend the stability limit and the robustness of the basic scheme. This technique was first introduced by Lerat ${ }^{16}$ in 1979 in conjunction with Lax-Wendroff type schemes. Later, in 1983, Jameson ${ }^{17}$ implemented it on the Runge-Kutta stepping scheme. In three dimensions we carried out the residual smoothing in the form,

$$
\left(1-\beta_{\xi} \nabla_{\xi} \Delta_{\xi}\right)\left(1-\beta_{\eta} \nabla_{\eta} \Delta_{\eta}\right)\left(1-\beta_{\zeta} \nabla_{\zeta} \Delta_{\zeta}\right) \tilde{R}=R
$$


where the residual $R$ includes the contribution of the variable time step and is defined by (25) and $\widetilde{R}$ is the residual after a sequence of smoothing in the $\xi$, $\eta$, and $\zeta$ directions with coefficients $\beta_{\xi}$, $\beta_{\eta}$, and $\beta_{\zeta}$.

For viscous calculations on highly stretched meshes the variable coefficient formulations of Martinelli ${ }^{10}$ and Swanson ${ }^{11}$ have proven to be robust and reliable. In the present paper, the expression for the variable coefficients $\beta$ of (30) has been modified to be used in three dimensions as follows,

$$
\begin{aligned}
& \beta_{\xi}=\operatorname{MAX}\left\{0, \frac{1}{4}\left[\left(\frac{C F L}{C F L^{*}} \frac{\lambda_{\xi}}{\lambda_{\xi}+\lambda_{\eta}+\lambda_{\zeta}} \phi_{\xi}\right)^{2}-1\right]\right\} \\
& \beta_{\eta}=\operatorname{MAX}\left\{0, \frac{1}{4}\left[\left(\frac{C F L}{C F L^{*}} \frac{\lambda_{\eta}}{\lambda_{\xi}+\lambda_{\eta}+\lambda_{\zeta}} \phi_{\eta}\right)^{2}-1\right]\right\} \\
& \beta_{\zeta}=\operatorname{MAX}\left\{0, \frac{1}{4}\left[\left(\frac{C F L}{C F L^{*}} \frac{\lambda_{\zeta}}{\lambda_{\xi}+\lambda_{\eta}+\lambda_{\zeta}} \phi_{\zeta}\right)^{2}-1\right]\right\}
\end{aligned}
$$

where the coefficients $\phi_{\xi}, \phi_{\eta}$, and $\phi_{\zeta}$ are the ones defined in eqs. (19), and $C F L$, and $C F L^{*}$ are the Courant numbers of the smoothed and unsmoothed scheme respectively. For the hybrid four-stage scheme we used $C F L=5$, and $C F L=2.5$.

\section{Multigrid}

This technique was developed in the beginning of the 1970s for the solution of elliptic problems ${ }^{18}$ and later was extended to time-dependent formulations 19,20.

The basic idea is to introduce a sequence of coarser grids and to use them to speed up the propagation of the fine grid corrections, resulting in a faster expulsion of disturbances. In this work we used the Full Approximation Storage (FAS) schemes of Brandt ${ }^{18}$ and Jameson 20.

Coarser, auxiliary meshes are obtained by doubling the mesh spacing and the solution is defined on them using a rule which conserves mass, momentum, and energy,

$$
\left(J^{1} Q^{(0)}\right)_{2 h}=\sum\left(J^{l} Q\right)_{h}
$$

where the subscripts refer to the grid spacing, and the sum is over the eight cells which compose the $2 h$ grid cell. Note that this definition coincides with the one used by Jameson when the reciprocal of the Jacobians are replaced with the cell volumes. To respect the fine grid 
approximation, forcing functions $P$ are defined on the coarser grids and added to the governing equations. So, after the initialization of $Q_{2 h}$ using eq.(32), forcing functions $P_{2 h}$ are defined as,

$$
P_{2 h}=\sum R_{h}\left(Q_{h}\right) \cdot R_{2 h}\left(Q_{2 h}^{(0)}\right)
$$

and added to the residuals $R_{2 h}$ to obtain the value $R_{2 h}^{*}$ which is then used for the stepping scheme.

$$
R_{2 h}^{*}=R_{2 h}\left(Q_{2 h}\right)+P_{2 h}
$$

This procedure is repeated on a succession of coarser grids and the corrections computed on each coarse grid are transferred back to the finer one by bilinear interpolations.

A V-type cycle with subiterations is used as a multigrid strategy. The process is advanced from the fine grid to the coarser one without any intermediate interpolation, and when the coarser grid is reached, corrections are passed back. One Runge-Kutta steps is performed on the $h$ grid, two on the $2 h$ grid, and three on all the coarser grids. It is our experience in cascade flow calculations that subiterations increase the robustness of the multigrid.

On each grid, the boundary conditions are treated in the same way and updated at every Runge-Kutta stage. For economy, the artificial dissipation model is replaced on the coarse grids with constant coefficient second-order differences.

The interpolations of the corrections introduce high frequency errors. In order to prevent those errors from being reflected in the eddy viscosity, turbulent quantities are updated after performing the stepping on the fine grid. On coarse grids the turbulent viscosity is evaluated by averaging the surrounding fine grid values.

\section{Grid Refinement}

A grid refinement strategy is used to provide a cost-effective initialization of the fine grid solution. This strategy is implemented in conjunction with multigrid to obtain a Full Multigrid (FMG) procedure. With the FMG method, the solution is initialized on a coarser grid of the basic grid sequence and iterated a prescribed number of cycles of the FAS scheme. The solution is then passed, by bilinear interpolations, onto the next, finer grid and the process is repeated until the finest grid level is reached. In the present paper we have introduced three levels of refinement with respectively two, three, and four grids.

\section{Computational Grid}

The three-dimensional grids are obtained by stacking two dimensional grids generated on the blade-to-blade surface ( $\xi, \eta$ plane). In the blade-to-blade projection, the grids are non-periodic $C$-type ones and are elliptically generated, controlling the grid spacing and orientation at the wall. The C-type structure has been chosen to model the blunt leading edge typical of turbine blading. 
The problem of grid skewness due to high stagger or large camber is addressed by allowing the grid to be non-periodic on the wake. This grid structure, recently introduced by the authors ${ }^{9}$ for two-dimensional applications, has proven to be effective in turbomachinery applications.

In the spanwise direction $(\zeta)$ a standard H-type structure has been adopted. Near the hub and tip walls geometric stretching is used for a specified number of grid points, after which the spanwise spacing remains constant.

\section{Applications and Discussion}

As applications of the procedure that has been described above, we used the TRAF3D code to predict the flow in an annular and in a linear cascade. The annular cascade is the one tested by Goldman and Seasholtz ${ }^{21,22}$, and experiments are available for configurations both with and without end wall contouring. The inlet boundary layer for this case is quite thin and the secondary flows are not extremely pronounced. On the contrary the large-scale linear cascade of Langston ${ }^{23,24}$ is a nice test case to investigate the code's capability of predicting the threedimensional features of the flow.

Calculations for these three blade passages will be presented in this section along with a grid sensitivity study.

\section{Goldman Annular Vane}

This annular vane was tested by Goldman ${ }^{21}$ at NASA Lewis with laser anemometer measurements. Pressure distribution in the blade passage, and details of the exit losses and angle spanwise variations made this test very interesting for code assessment.

In a previous work of ours ${ }^{9}$ we carried out a two-dimensional grid dependency study in order to figure out the mesh requirements necessary to obtain a space-converged calculation, especially for skin friction and heat transfer predictions. Those results can be extrapolated to the three-dimensional case but the resulting number of grid points is quite large: nearly half million points. In three-dimensional applications, the memory and time requirements can be large, even for a modern supercomputer, so a grid dependency study can be useful in optimizing the mesh size. We introduced three grids of respectively $97 \times 17 \times 25,127 \times 25 \times 49$, and $177 \times 33 \times 65$ grid points. The fine mesh spacing at the wall is $2 \times 10^{-4}$ axial chord in the the blade-to-blade direction and $5 \times 10^{-4}$ in the spanwise one. Using those spacings, the $y^{+}$at the wall is roughly less than two when the exit Reynolds number is about one million. On the medium and coarse grids the wall spacings are respectively two times and four times the fine grid ones. On the three meshes there are respectively 33,49 , and 65 points on the suction surface of the airfoil. A threedimensional view of the fine grid for the Goldman cascade without end-wall contouring is given in fig. 1(a). The low level of skewness obtained by stacking non-periodic C-type grids is evident.

The convergence of the root mean square of the residuals of the continuity equation is given in fig. 2(a). This calculation refers to the fine mesh (380,000 grid points) and requires about 45 minutes on the NASA Lewis Cray Y-MP. If we assume we have reached a good level of convergence when the residuals have dropped four decades, the medium mesh $(156,000$ grid 
points) takes 15 minutes, while about 6 minutes are needed for the coarse mesh calculation (4l,000 grid points).

For this test case, the inlet flow is axial and the exit isentropic Mach number at the hub is about 0.809 . Figures 2(b), (c), and (d) show the experimental and computed isentropic surface Mach number distribution near the hub, at midspan, and near the tip. All the grids correctly predict the distribution, the slight underestimation of the coarse grid with respect to the medium and fine ones is mostly due to the higher level of predicted losses.

In ref. 21 Goldman measured the spanwise distributions of the aftermixed pressure, exit flow angle, total pressure loss coefficient, and energy loss coefficient at $1 / 3$ axial chord away from the trailing edge. The predicted distribution of these quantities is compared to experiments in fig. 3. The agreement is very good on the whole. The radial distribution of the static pressure is accurately reproduced (see fig. 3(a)), while the exit angle is qualitatively the same on all the grids but with a smoother distribution with respect to experiments (fig.3(b)). From the plots of total pressure and energy loss coefficients of figs. 3(c) and (d) we can draw some interesting conclusions. In the coarse grid, with only 25 points in the hub to tip direction, the distribution of the losses is only roughly captured. We need at least 49 points to have a spanwise loss prediction which is nearly space-converged. Coarse grid losses are also twice as large as the experiment at midspan, thus confirming the indication of the two-dimensional analysis ${ }^{9}$ which suggested the use of $y^{+}$near the unity. Both the medium and fine grids predict the losses well, except near the tip, where the boundary layer is thicker. The kink in the losses is computed closer to the wall than experiments indicate.

The position of the horseshoe vortices near the hub and tip is given in fig. 4. The enlargements show the counter-rotating comer vortices.

The lift of the passage vortex on the suction side of the airfoil is evident from the particle trace near the wall of fig.5(a). The effect is more pronounced near the tip because of the thicker inlet boundary layer.

\section{Goldman Annular Vane With Contoured End Wall}

Figure 1(b) shows the $177 \times 33 \times 65$ computational mesh for the Goldman vane with an sshape contoured end wall at the hub after midchord. Some details of the calculation for this case are summarized in figs. 5(b) and 6. The exit isentropic Mach number at the hub is now about 0.695 . The computed surface isentropic Mach number agrees well with experiments ${ }^{22}$ near the hub, at midspan, and near the tip. On the hub the presence of the contoured end wall causes a stronger pressure gradient between pressure and suction sides as a result the flow lift of soner on the suction side as shown by the simulated oil flow-trace of fig. 5(b).

\section{Langston Low-Speed Linear Cascade}

Langston et al. ${ }^{23}$ in 1976 made detailed measurements in a low-speed, large-scale plane turbine cascade. The work included experimental visualizations of the three-dimensional nature of the flow. The flow exhibits important three-dimensional separation on both airfoil surface and end wall.

Even though the flow conditions of this test are at very low speed and not amenable to calculations using compressible flow codes, the Langston cascade is quite often used in three- 
dimensional code validation. The $177 \times 33 \times 65$ grid we used is depicted in fig. 7 . Graziani 24 took these visualizations of the flow for two different inlet boundary layer thicknesses. As the aim of this study is to investigate the capability of reproducing the three-dimensional features of the flow, we are reporting calculations for the thick inlet boundary layer. Figure 8 compares the predicted and measured end wall limiting streamlines. The structure of the flow, as well as the position of the saddle point, are in good agreement with experiments. The fact that the computed saddle point is ahead of the experiment is common to several calculations (i.e. refs. 5 and 7). In our opinion this discrepancy can be partially attributed to the difficulty of having very similar inlet boundary layers in the calculations as in the experiments. On the suction surface of the airfoil, the particle traces of fig. 9 and the experimental limiting streamlines clearly demonstrate the code's capability in predicting the three-dimensional flow separation.

\section{Conclusions}

The central-difference, finite-volume scheme with eigenvalue scaling for artificial dissipation terms, variable-coefficient implicit smoothing, and full multigrid has been extended to predict three-dimensional viscous cascade flows. We report the comparisons of calculations with experiments for the Goldman annular cascade with and without end wall contouring, and for the Langston cascade. Good overall predictions can be obtained with the Baldwin-Lomax turbulence model both in terms of pressure distribution and loss coefficients, for the cases studied. The grid dependency study has also been conducted to determinate the grid spacing necessary to capture fine details of the three-dimensional viscous flows. With these accelerating strategies, detailed three-dimensional viscous solutions can be obtained for a reasonable fine grid in less than one hour on a modern supercomputer.

\section{Acknowledgements}

The first author would like to express his gratitude to ICOMP and NASA for providing support, facilities, and computer time for this work. Thanks are also due to Prof. Eli Turkel for the useful discussions on extending the scheme to 3D. Further thanks are due to Prof. Sergio S. Stecco of the University of Florence.

\section{References}

1. Chima, R. V., and Yokota, J. W., "Numerical Analysis of Three-Dimensional Viscous Internal Flows," NASA TM 100878, 1988.

2. Weber, K. F., and Delaney, R. A., "Viscous Analysis of Three-Dimensional Turbomachinery Flows on Body Conforming Grids Using an Implicit Solver," ASME paper No. 91-GT-205, 1991. 
3. Hah, C., "Numerical Study of Three-Dimensional Flow and Heat Transfer Near the Endwall of a Turbine Blade Row, " AIAA paper No. 89-1689, 1989.

4. Nakahashi, K., Nozaki, O., Kikuchi, K., and Tamura, A., "Navier-Stokes Computations of Two- and Three- Dimensional Cascade Flowfields, " AlAA Journal of Propulsion and Power, Vol. 5, No. 3, May-June 1989, pp. 320-326.

5. Choi, D., and Knight, C. J., "Computation of Three-Dimensional Viscous Liner Cascade Flows, " AIAA Journal, Vol. 26, No. 12, December 1988, pp. 1477-1482.

6. Dawes, N. W., "The Simulation of Three-Dimensional Viscous Flow in Turbomachinery Geometries Using a Solution-Adaptive Unstructured Mesh Methodology, " ASME paper No. 91-GT-124, 1991.

7. Subramanian, S. V., and Bozzola, R., "Numerical Simulation of Three-Dimensional Flow Fields in Turbomachinery Blade Rows Using the Compressible Navier-Stokes Equations, " AIAA paper No. 87-1314, 1987.

8. Amone, A., and Swanson, R. C., "A Navier-Stokes Solver for Cascade Flows, " NASA CR No. $181682,1988$.

9. Amone, A., Liou, M-S, and Povinelli, L. A., "Transonic Cascade Flow Calculations Using Non-Periodic C-Type Grids, "Computational Fluid Dynamics Symposium on Aeropropulsion, NASA Lewis Research Center, Cleveland, April, 1990.

10. Martinelli, L. and Jameson, A. "Validation of a Multigrid Method for the Reynolds Averaged Equations, " AIAA paper No. 88-0414, 1988.

11. Swanson, R. C., and Turkel, E., "Artificial Dissipation and Central Difference Schemes for the Euler and Navier-Stokes Equations., " AIAA paper No. 87-1107, 1987.

12. Baldwin, B. S., and Lomax, H. "Thin Layer Approximation and Algebraic Model for Separated Turbulent Flows, " AIAA paper 78-257, 1978.

13. Jameson, A., Schmidt, W., and Turkel, E., "Numerical Solutions of the Euler Equations by Finite Volume Methods Using Runge-Kutta Time-Stepping Schemes, " AIAA paper No. 811259, 1981.

14. Pulliam, T. H., "Artificial Dissipation Models for the Euler Equations, "AIAA Journal, Vol. 24, No. 12, December 1986, pp. 1931-1940.

15. Amone, A., and Stecco, S. S., "Inviscid Cascade Flow Calculations Using a Multigrid Method, " ASME paper No. 89-GT-22, 1989.

16. Lerat, A., "Une Classe de Schemas aux Differences Implicites Pour les Systemes Hyperboliques de Lois de Conservation, "Comptes Rendus Acad. Sciences Paris, Vol. 288 A, 1979.

17. Jameson, A., "The Evolution of Computational Methods in Aerodynamics, " J. Appl. Mech., Vol. 50, 1983.

18. Brandt, A., "Multi-Level Adaptive Computations in Fluid Dynamics, " AIAA paper No. 79$1455,1979$.

19. Ni, R-H, "A Multiple-Grid Scheme for Solving the Euler Equations, " AIAA paper No. 811025, 1981.

20. Jameson, A., "Transonic Flow Calculations, " MAE Report 1651, MAE Department, Princeton University, July 1983. 
21. Goldman, L. J., and Seasholtz, R., "Laser Anemometer Measurements in an Annular Cascade of Core Turbine Vanes and Comparison With Theory, "NASA TP No. 2018, 1982.

22. Goldman, L. J., and Seasholtz, R., "Three Component Laser Anemometer Measurements in an Annular Cascade of Core Turbine Vanes With Contoured End Wall, " NASA TP No. 2846, 1988.

23. Langston, L. S., Nice, M. L., and Hooper, R. M., "Three-Dimensional Flow Within a Turbine Cascade Passage, " ASME paper No. 76-GT-50, 1976.

24. Graziani, R. A., Blair, M. F., Taylor, J. R., and Mayle, R. E., "An Experimental Study of Endwall and Aiffoil Surface Heat Transfer in a Large Scale Turbine Blade Cascade, " ASME paper No. 79-GT-99, 1979. 

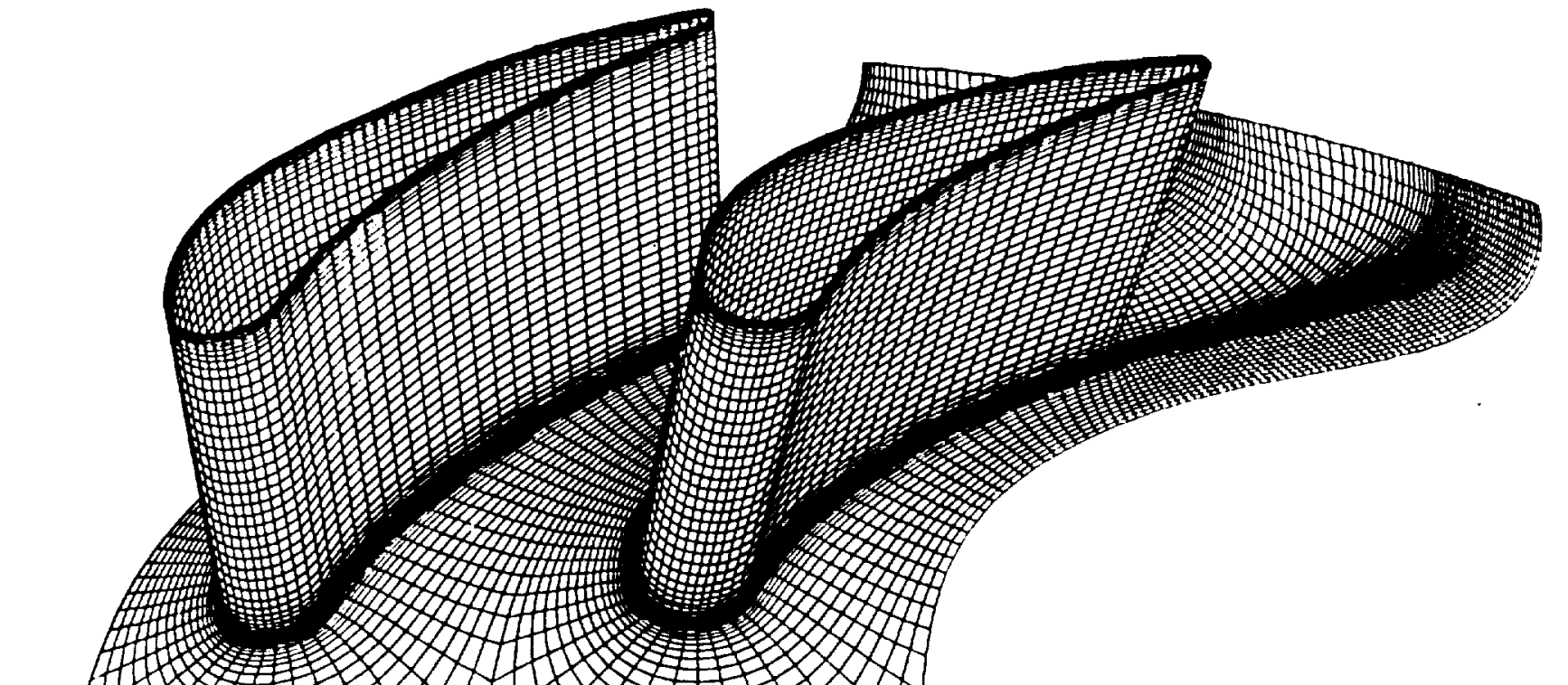

a) Without contoured end wall
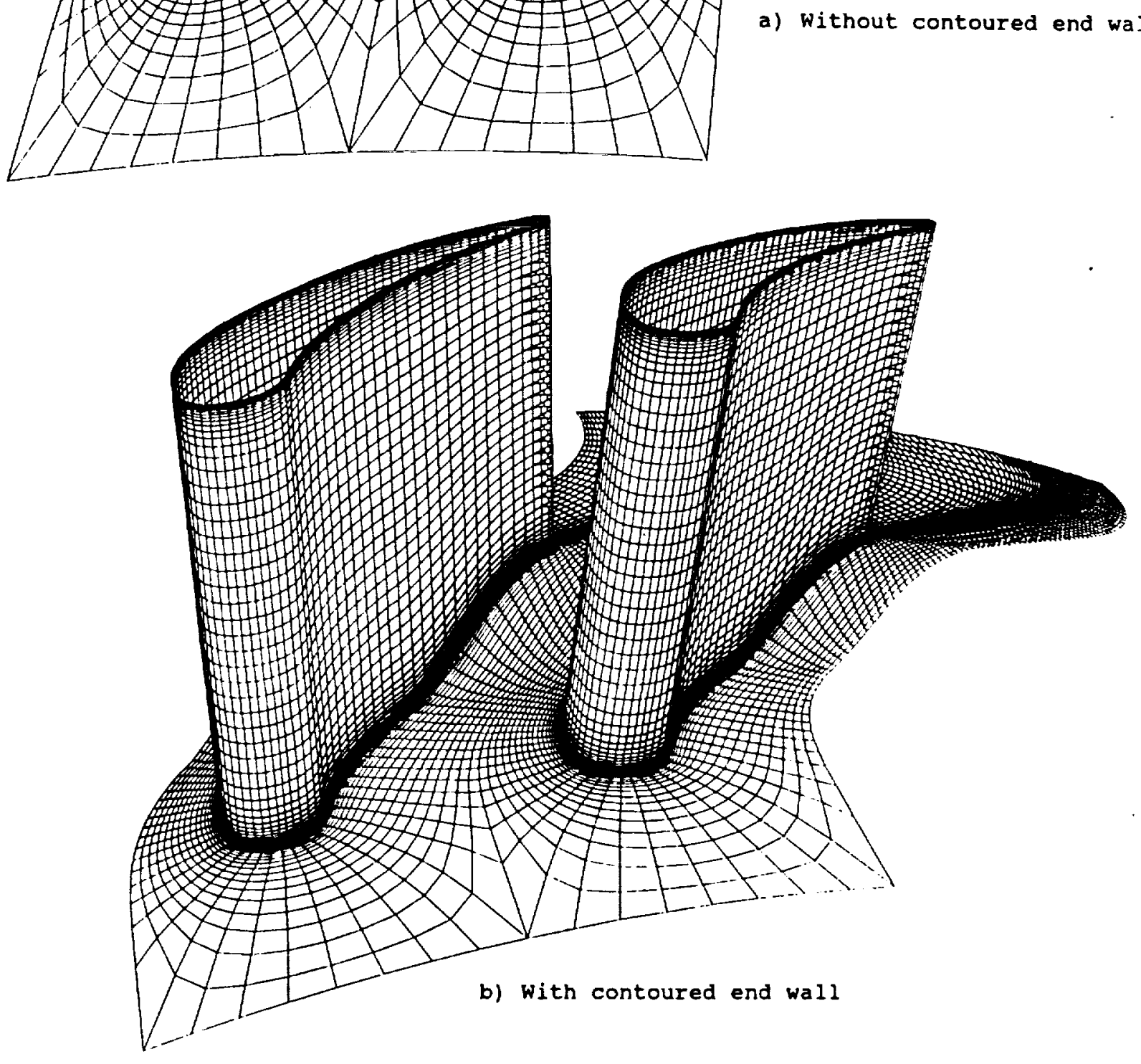

Fig. $1177 \times 33 \times 65$ Computational grids for the Goldman annular vanes with and without contoured end walls. 


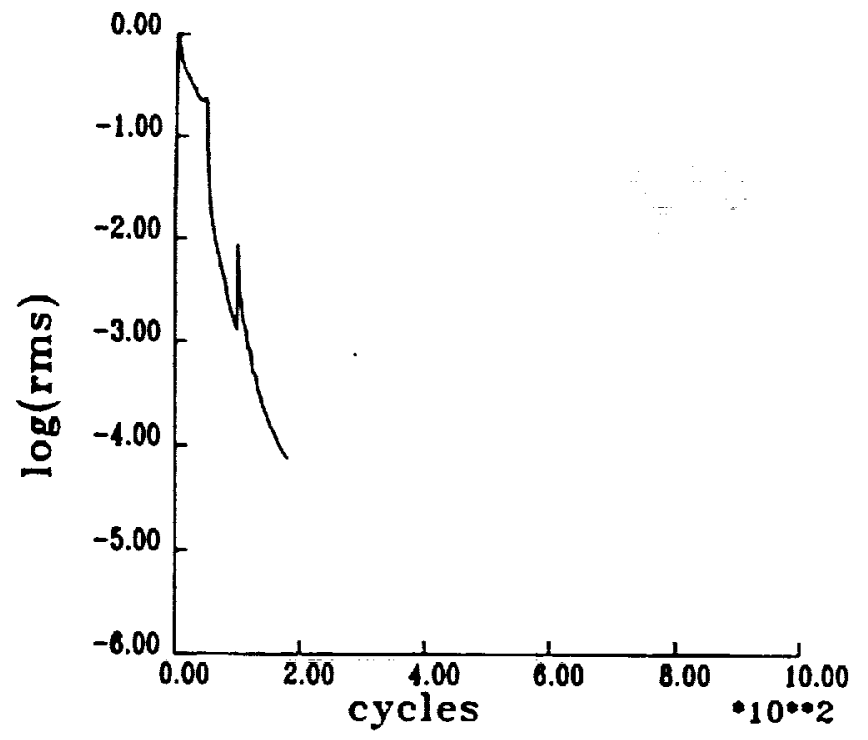

a) Convergence history

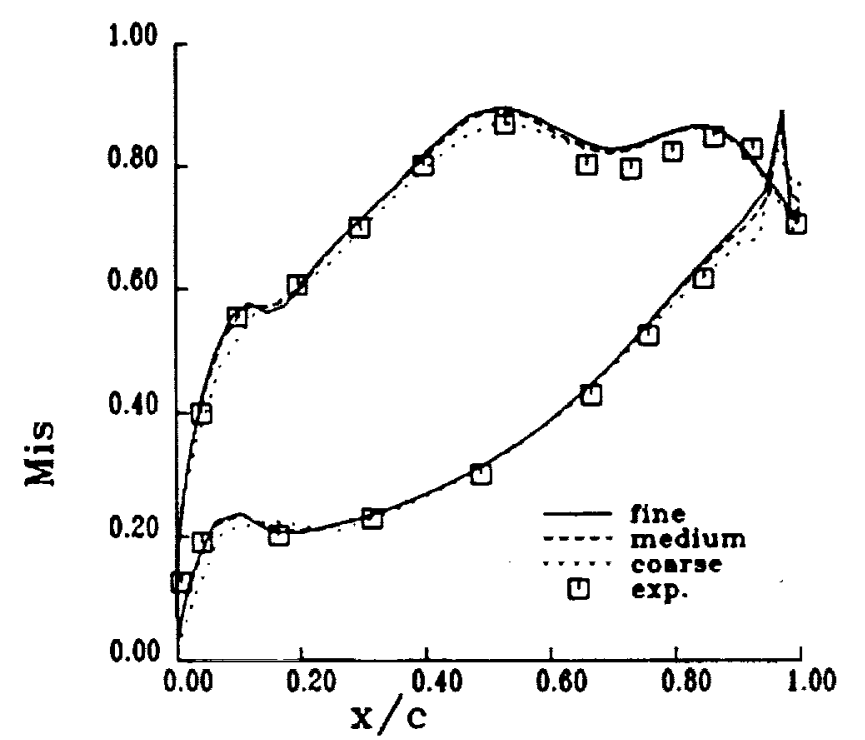

c) Isentropic Mach number distribution at midspan

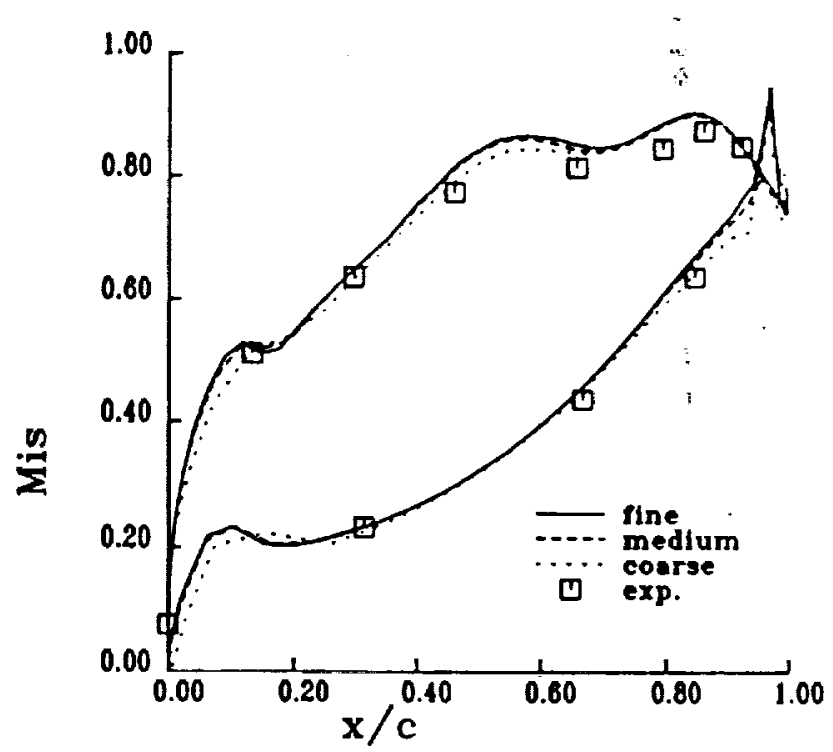

b) Isentropic Mach number distribution near hub

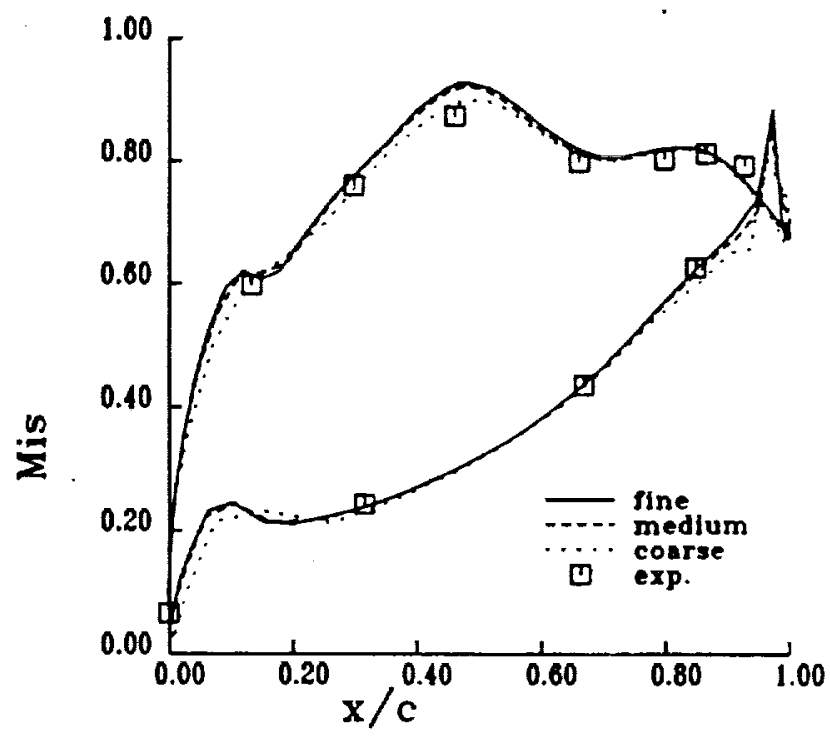

d) Isentropic Mach number distribution near tip

Fig. 2 Goldman annular vane without contoured end wall. 


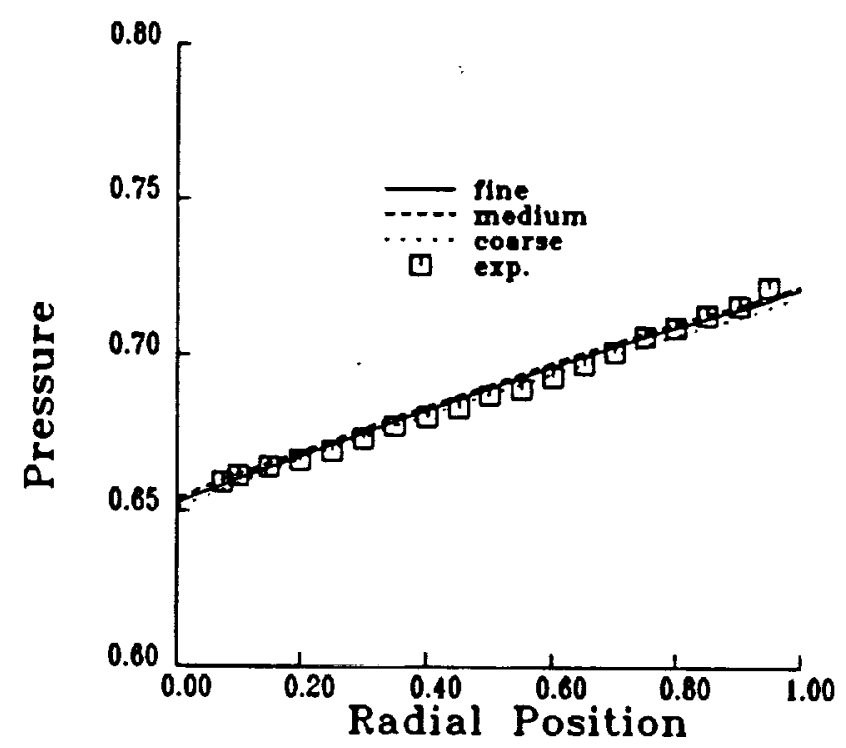

a) Pressure

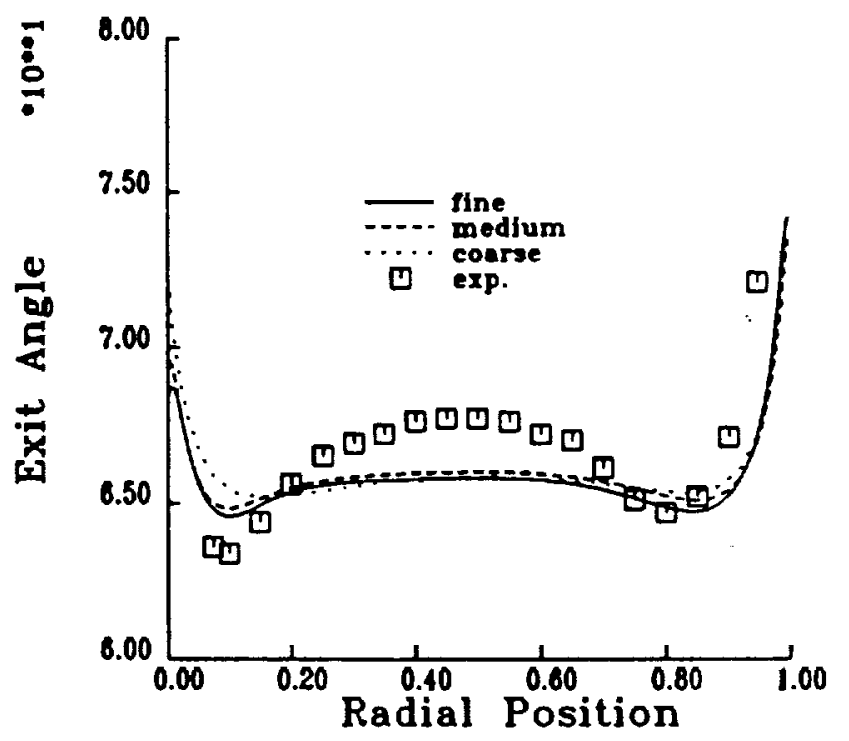

b) Exit angle
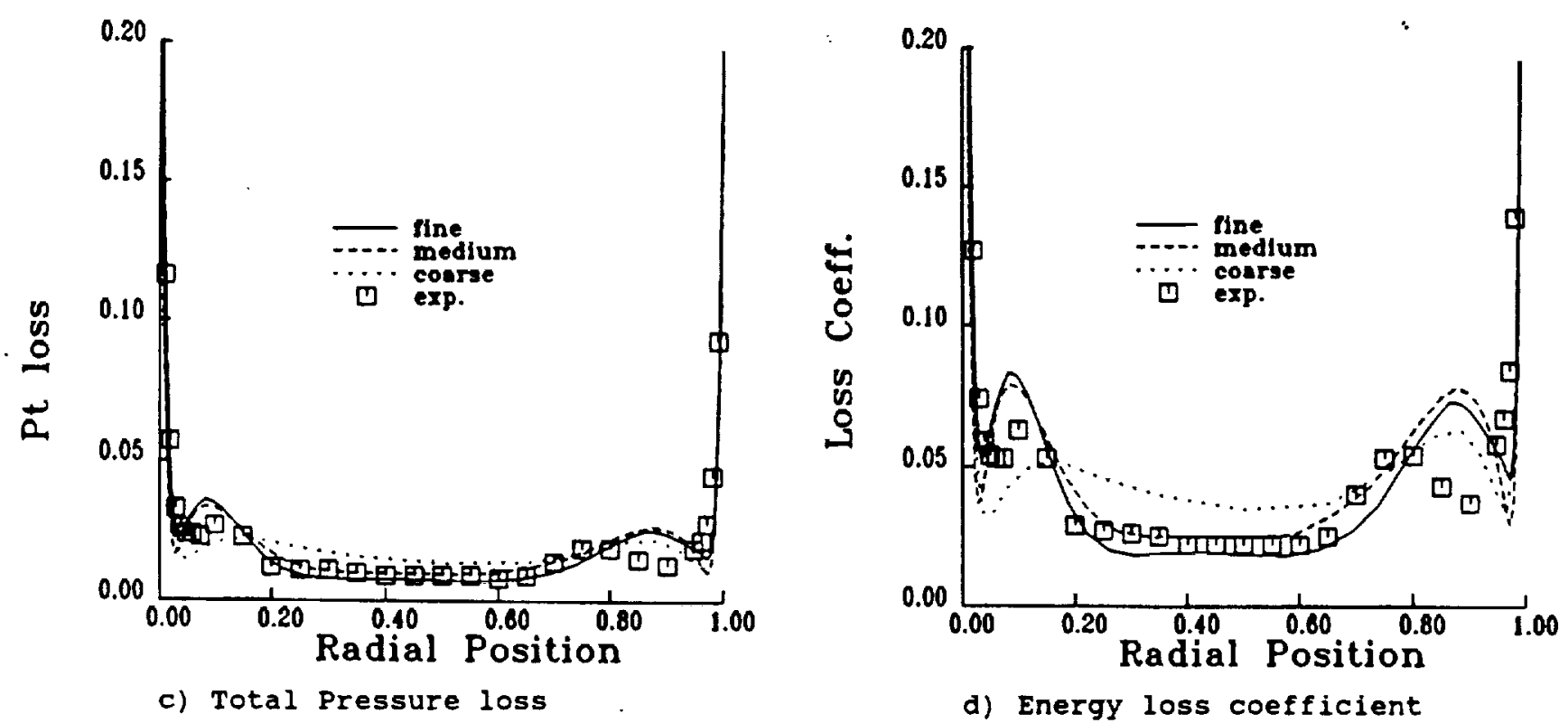

d) Energy loss coefficient

Fig. 3 Goldman annular vane without contoured end wall. Aftermixed spanwise distribution of pressure, exit angle, and loss coefficients at $1 / 3$ axial chord away from trailing edge. 


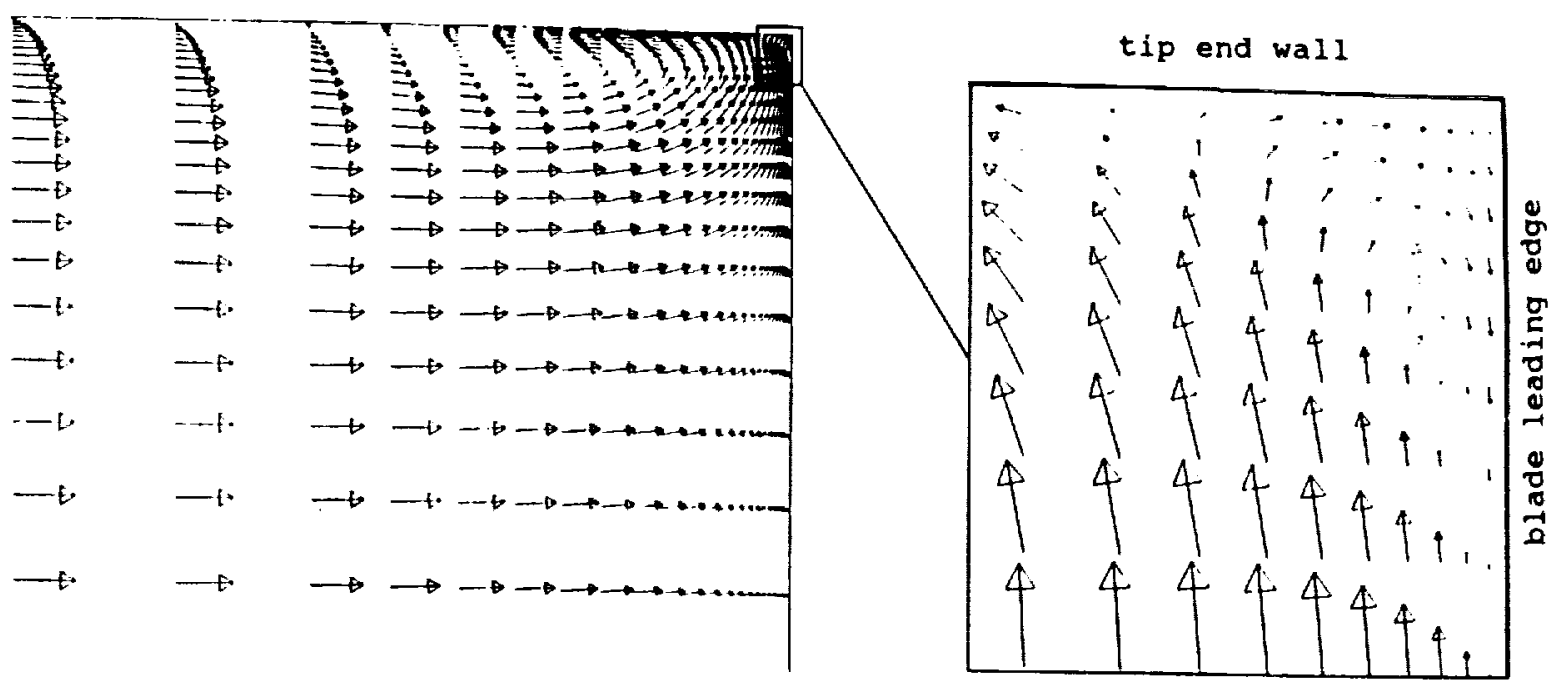

a) Horseshoe vortex and counter-rotating corner vortex near tip

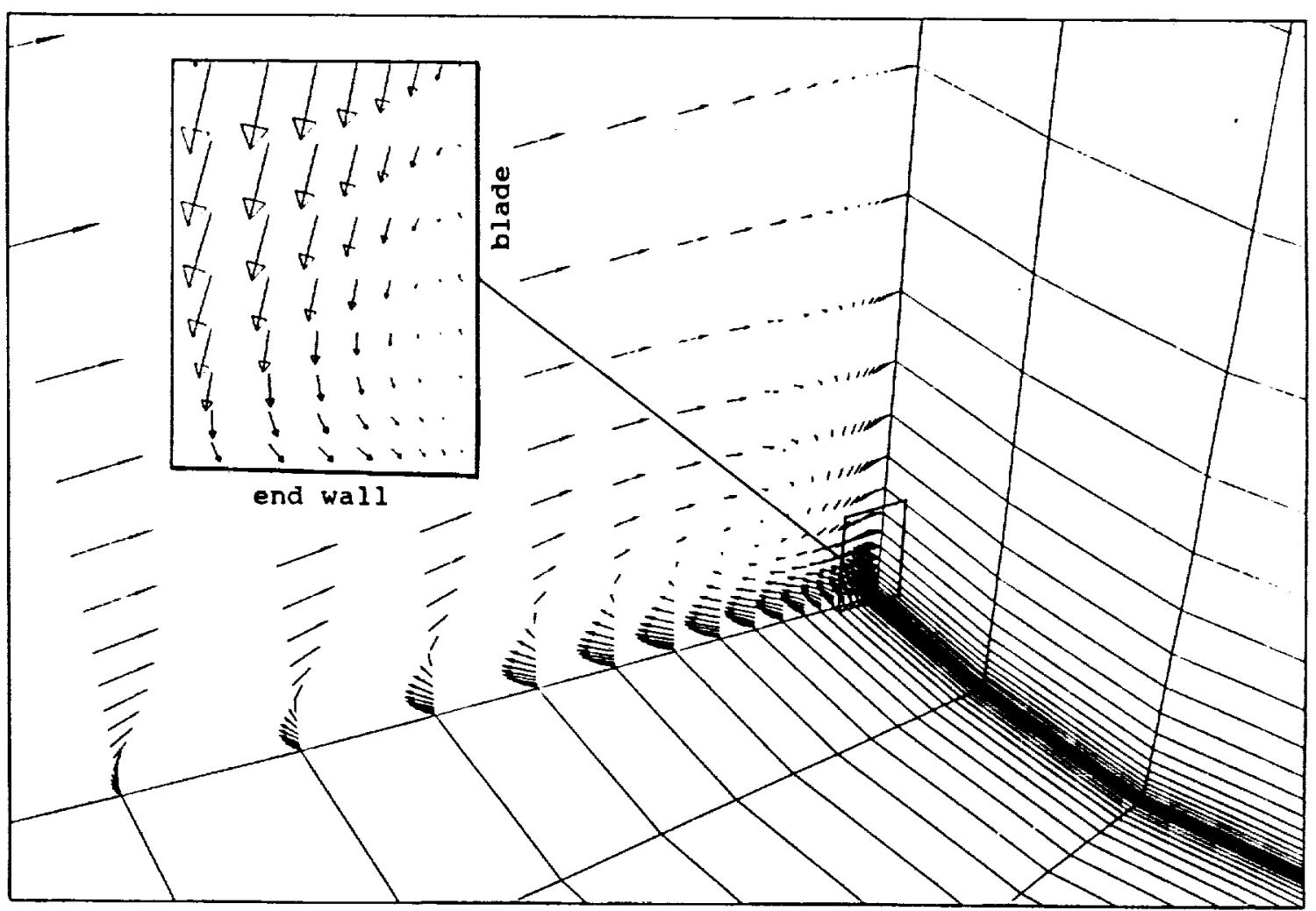

b) Horseshoe vortex and counter-rotating corner vortex near hub

Fig. 4 Details of the horseshoe vortices near tip and hub. 


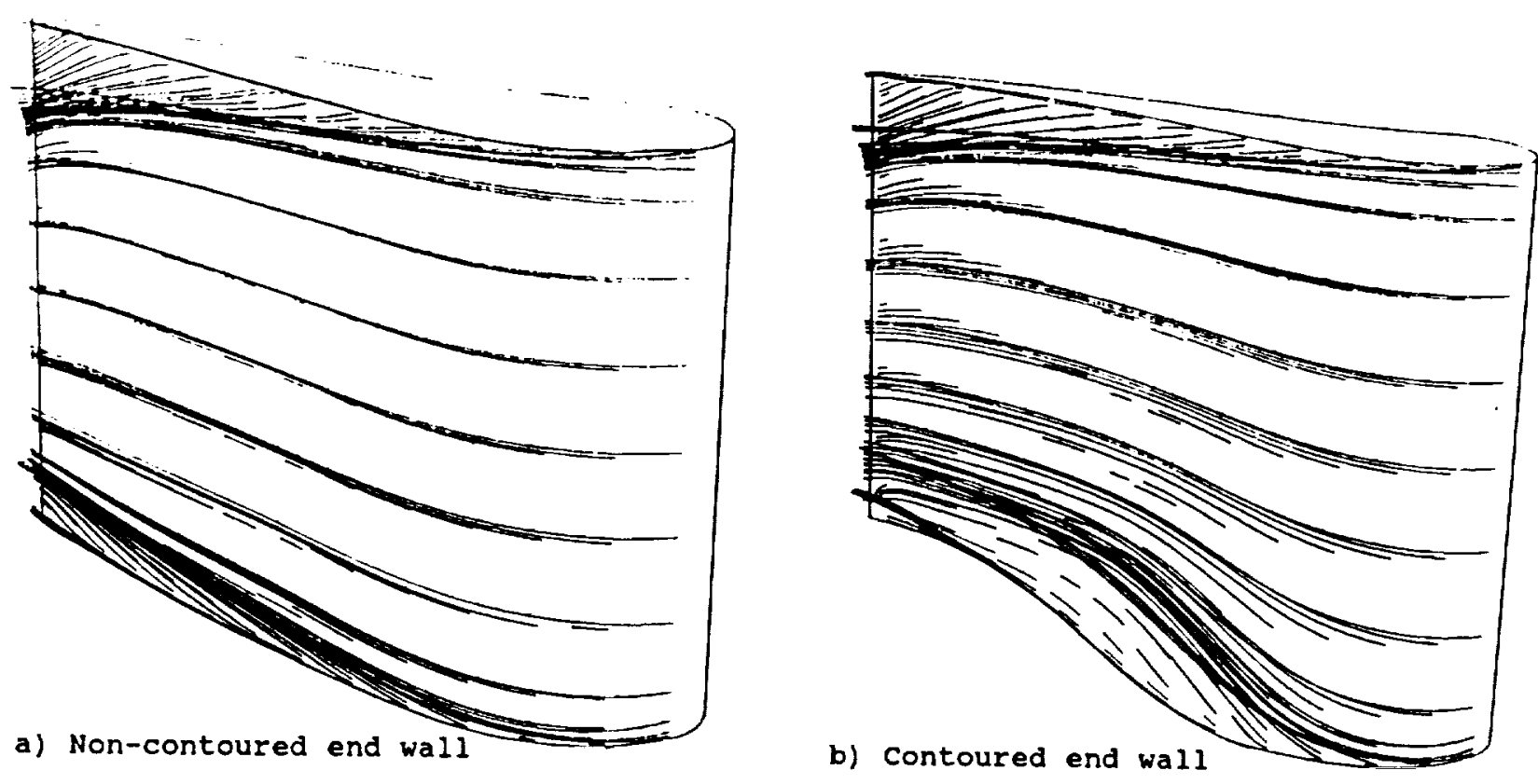

Fig. 5 Particle traces on the suction side of the Goldman annular vane,
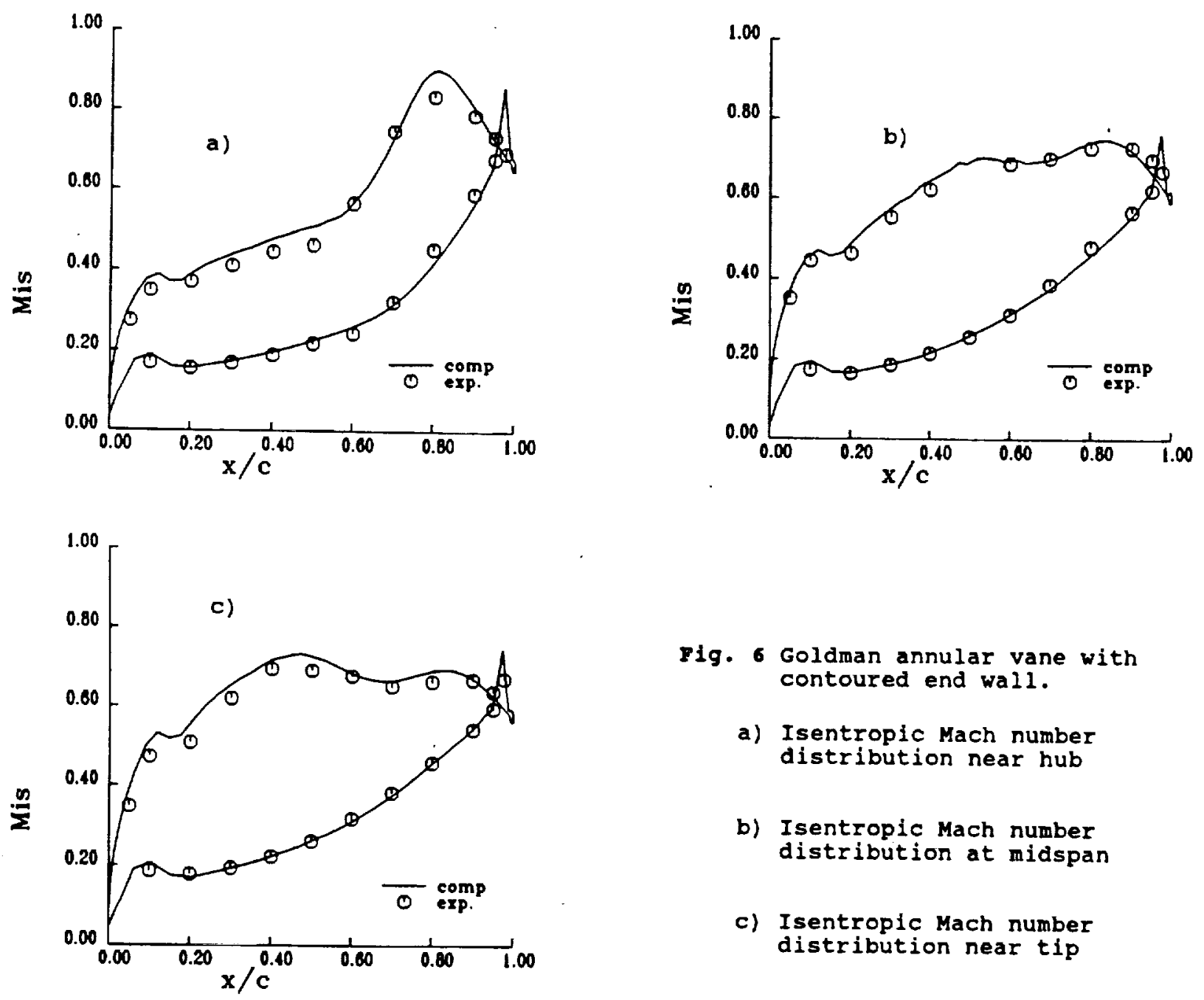

Fig. Goldman annular vane with contoured end wall.

a) Isentropic Mach number distribution near hub

b) Isentropic Mach number distribution at midspan

c) Isentropic Mach number distribution near tip 


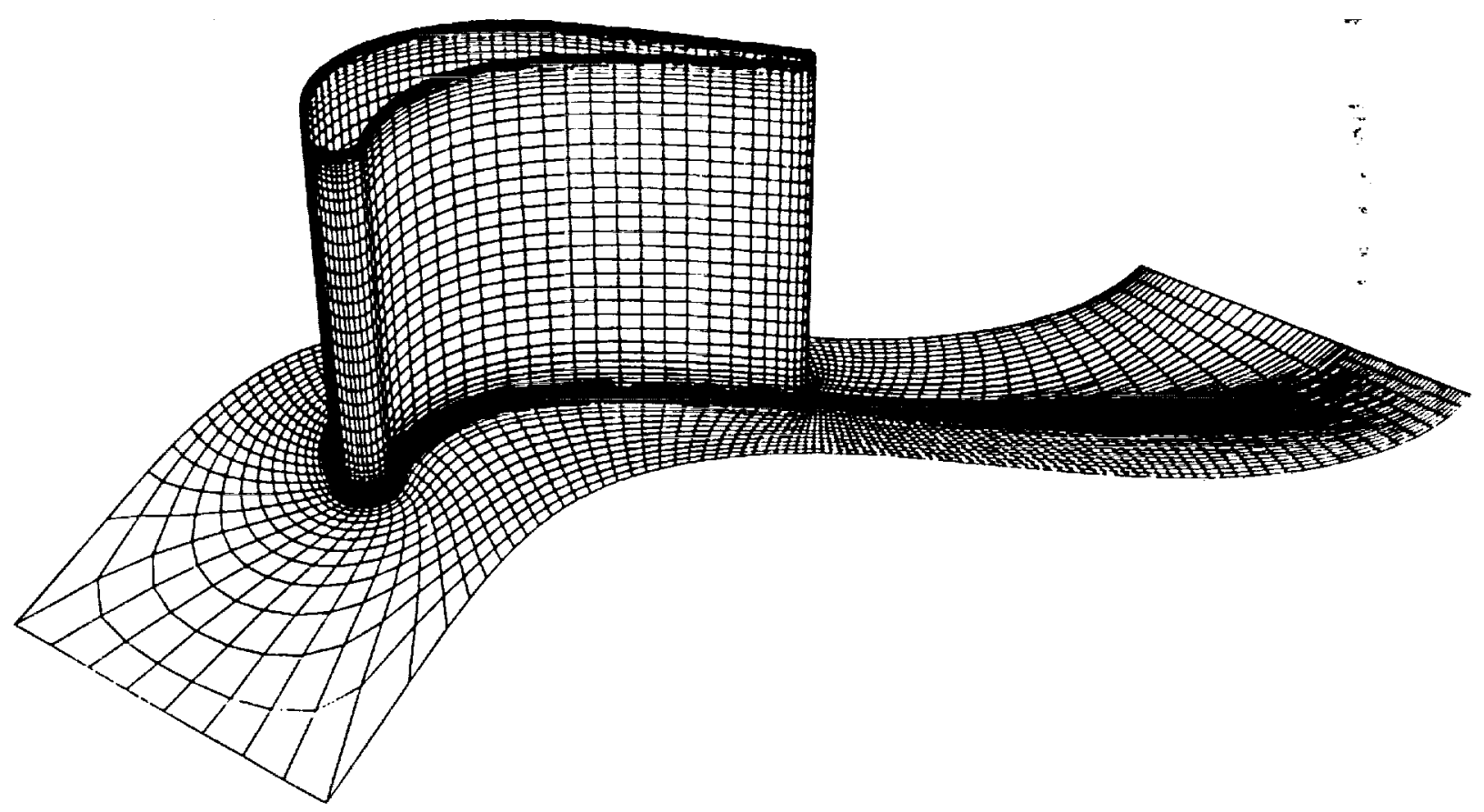

Fig. $7177 \times 33 \times 65$ computational grid for the Langston cascade.
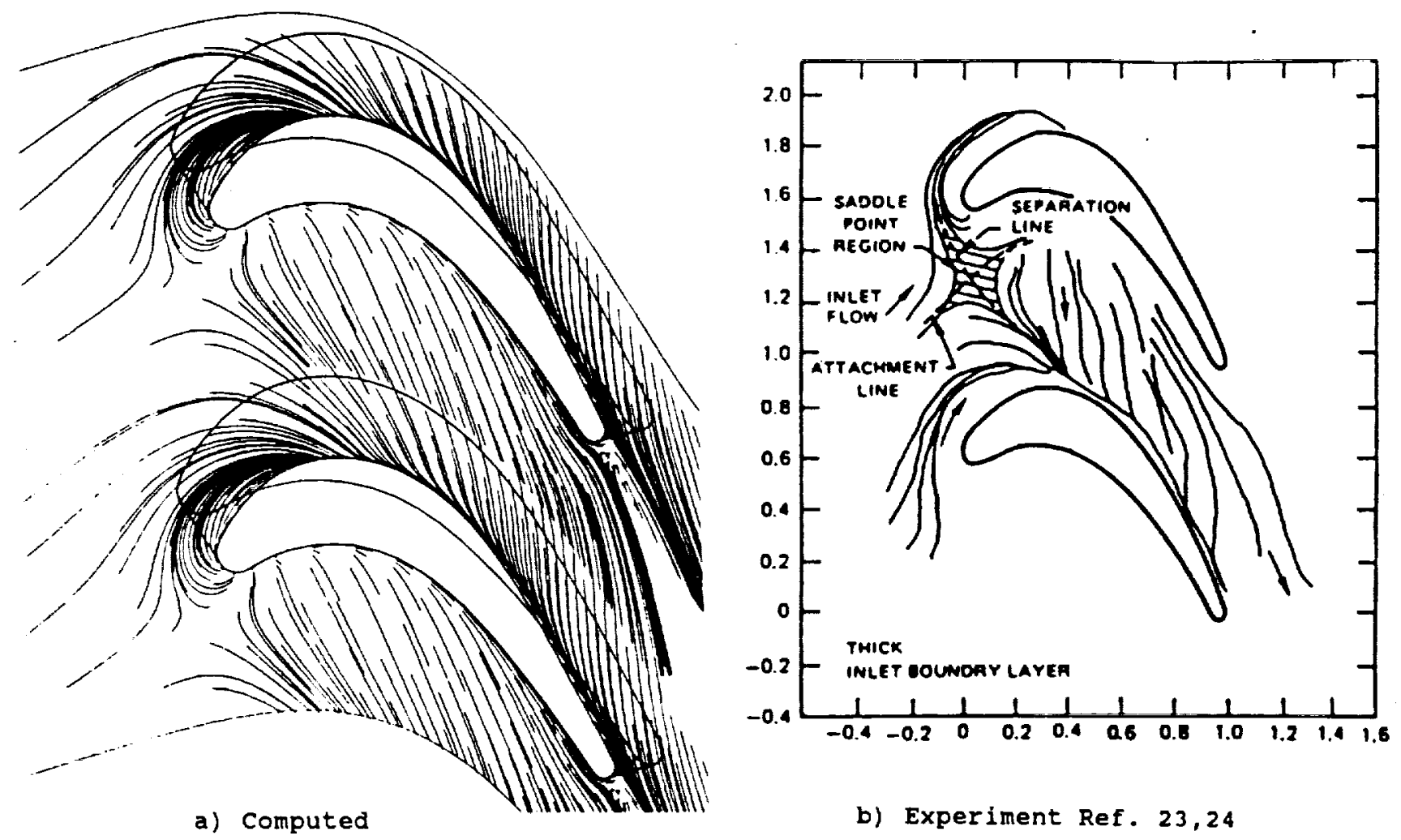

Fig. 8 Computed and experimental end wall limiting stream lines. 


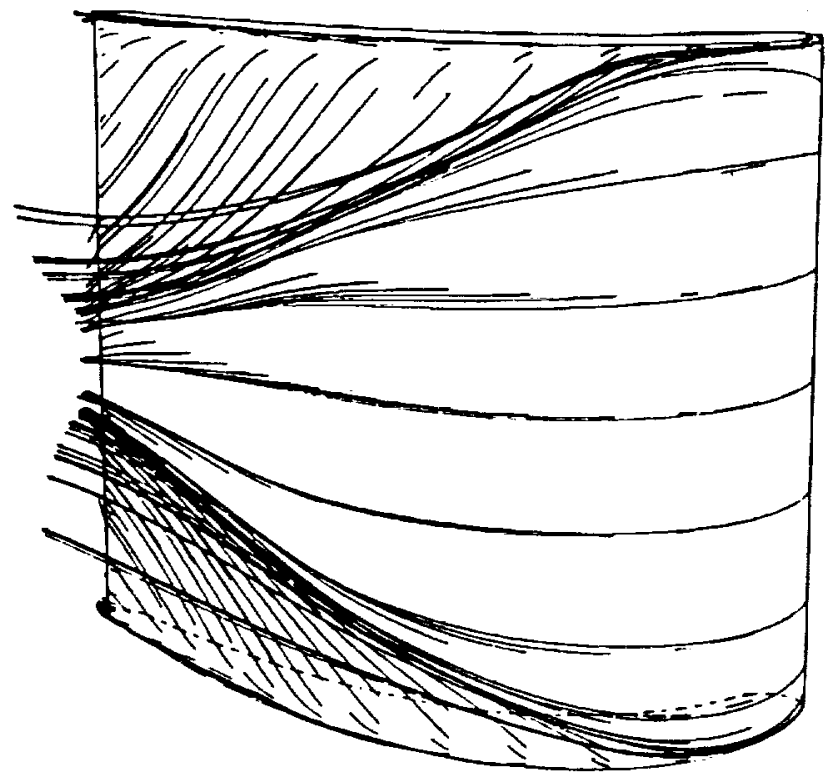

a) Computed

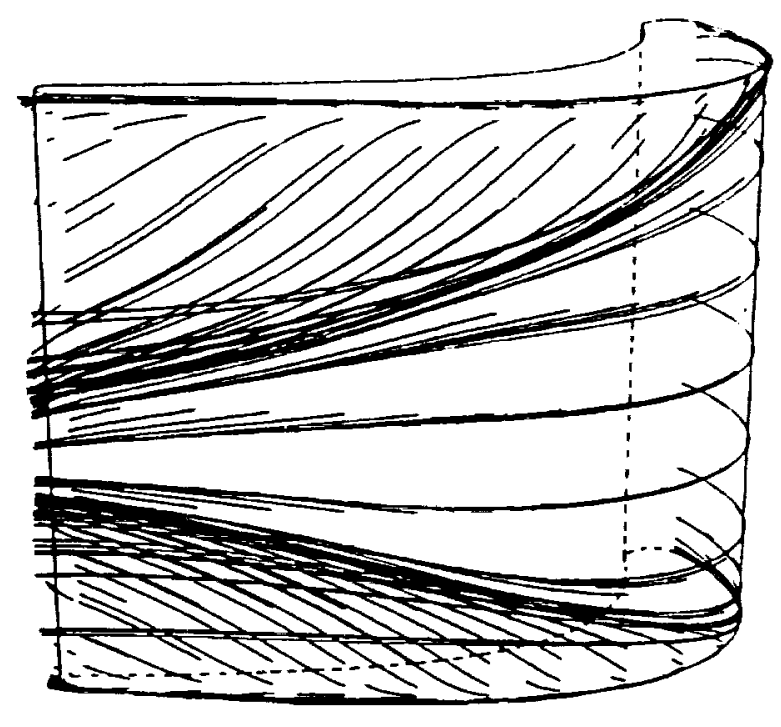

b) Computed

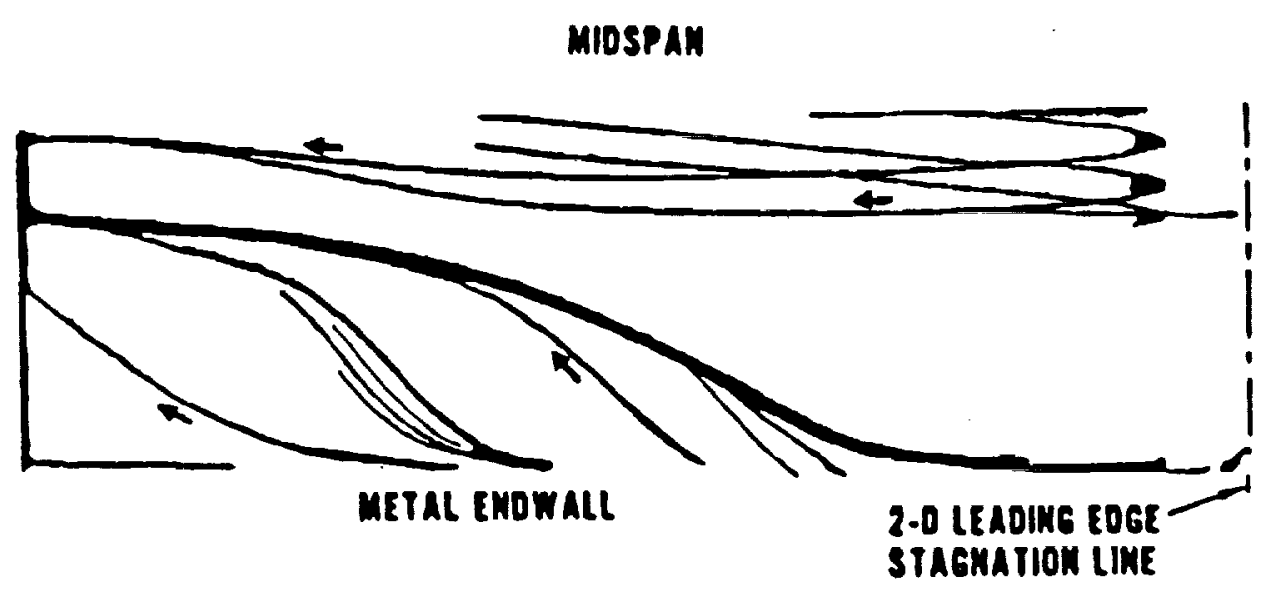

c) Experiments Ref. 23,24

Fig. I Langston cascade. Computed and experimental limiting streamlines on suction side. 
Public reporting burden for this collection of information la estimated to averepe 1 hour per response, inciuding the fime for reviewing instructions, searching existing data soufces gathering and maintuinlng the data neoded, and completing and reviewing the collection of inlormation. Send comments regarding this burden estimale or any other aspect of this coltection of information, induding alpgestions for reducing this burden, to Weshington Headquariers Sorvices, Directorate tor information Operations and Reports, 20503 .

1. AGENCY USE ONLY (Leave blank) 2. REPORT DATE 3. REPORT TYPE AND DATES COVERED

\section{TITLE AND SUBTITLE}

October 1991

Technical Memorandum

Multigrid Calculation of Three-Dimensional Viscous Cascade Flows 5. FUNDING NUMBEAS

8. AUTHOR(S)

WU $-505-62-21$

A. Amone, M.-S. Liou, and L.A. Povinelli

7. PERFoRMINO OROANIZATION NAME(S) AND ADDRESS(ES)

8. PERForming oRganIZATION REPORT NUMBEF

National Aeronautics and Space Administration

Lewis Research Center

$E-6587$

Cleveland, Ohio 44135-3191

9. SPONSORING/MONITORING AGENCY NAMES(S) AND ADDRESS(ES)

10. SPONSORING/MONITORING AGENCY REPORT NUMBER

National Aeronautics and Space Administration

Washington, D.C. 20546-0001

NASA TM - 105257

ICOMP -91-18

\section{SUPPLEMENTARY NOTES}

A. Arnone, University of Florence, Florence, Italy and Institute for Computational Mechanics in Propulsion, Lewis Research Center, Cleveland, Ohio (work funded under Space Act Agreement C-99066-G). M.-S. Liou and L.A. Povinelli, NASA Lewis Research Center. Space Act Monitor: Louis A. Povinelli, (216) 433-5818.

12. DISTAIBUTION/AVAILABILITY STATEMENT 12b. DISTRIBUTION CODE

Unclassified - Unlimited

Subject Category 64

13. ABSTRACT (Moximum 200 words)

A three-dimensional code for viscous cascade flow prediction has been developed. The space discretization uses a cell-centered scheme with eigenvalue scaling to weigh the artificial dissipation terms. Computational efficiency of a four-stage Runge-Kutta scheme is enhanced by using variable coefficients, implicit residual smoothing, and a fullmultigrid method. The Baldwin-Lomax eddy-viscosity model is used for turbulence closure. A zonal, non-periodic grid is used to minimize mesh distortion in and downstream of the throat region. Applications are presented for an annular vane with and without end wall contouring, and for a large-scale linear cascade. The calculation is validated by comparing with experiments and by studying grid dependency.

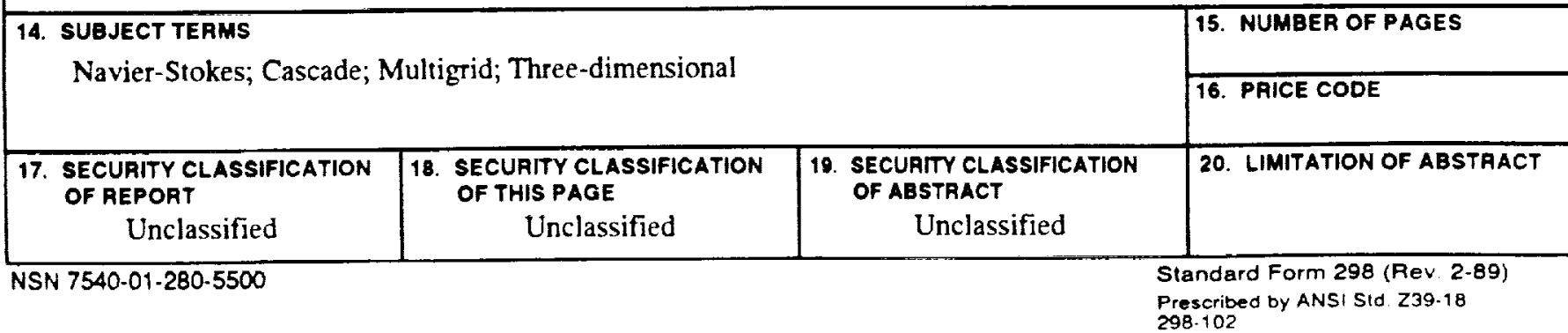

